\title{
CHROMOSOMAL ANALYSIS OF CATFISH, CLARIAS GARIEPINUS (L.)
}

\author{
Yogesh Bhute ${ }^{1 *}$, Shivkumar Chauhan ${ }^{3}$ and V.V. Baile ${ }^{2}$ \\ ${ }^{1}$ Department of Zoology, Sindhu Mahavidyalaya, Panchpaoli, Nagpur 440017 \\ ${ }^{2}$ Department of Zoology, Nagpur University, Nagpur (M.S.), India-440 033 \\ ${ }^{3}$ Pregen, Preventive Genetics Diagnostic Centre, Ajni, Nagpur (M.S.) India \\ 444015.
}

Communicated: 13.02 .21

\begin{abstract}
:
Clarias gariepinus is an African catfish, commonly found in most of the regions of India. This is a fast growing and hardy fish as compared to other indigenous catfishes. In the present investigation, chromosome number and karyotyping of C. gariepinus are performed. By using phytohemagglutinin (PHA) activated whole blood cell culture, metaphase chromosomes were isolated and studied. Metaphase shows that it has $2 \mathrm{n}=54$ number of chromosomes. The chromosomes are further classified as 08 metacentric, 22 submetacentric, 14 subtelocentric and 10 telocentric. This study is important for knowing genomic constitution of this fish as well as it is useful for hybridization with other indigenous catfishes.
\end{abstract}

Keywords: Chromosomes, Karyotype, Clarias gariepinus

\section{INTRODUCTION:}

In inland water bodies, carps and catfishes dominate the production but most of the work related to genetics is carried out on carps only (Khuda-Bukhsh, 1980; Manna, 1983) and among the catfishes cytogenetics work on Clarias batrachus and Heteropneustes fossilis (DuttaMunshi and Hughes, 1992) is reported. For the viable hybrid progeny, compatibility of diploid (2n) number of chromosomes of proposed species is the most important factor. Clarias gariepinus, selected for the present study is fast growing fish and is a good model for fish hybridization. Karyotype study of fish is also used in taxonomic arrangement in classification and identification. Karyological studies in fish have shown potentials in increasing knowledge in the fields of genetics, taxonomy, evolution, systematics, mutagenesis, environmental toxicology and aquaculture (Kligerman and Bloom, 1977; Cucchi and Baruffaldi, 1990) In other animals like aves, 211 species belonging to 99 genera karyologically are known (Mohanty, 2004) but very less information is available on chromosomes of many of the fish species in general including the fish Clarias gariepinus. To elucidate the knowledge of chromosomal number and pattern, studies on this fish are carried out.

\section{MATERIAL \& METHODS:}

Fish were collected from the local market, acclimatized in laboratory conditions for one week, anesthetized with2-phenoxyethanol and blood was collected from heart by puncturing with needle syringe coated with heparin. Blood was centrifuged in transparent centrifuge tube for $15 \mathrm{~min}$. at $400 \mathrm{~g}$. Plasma was carefully removed without disturbing the interface. Interface was collected with syringe or Pasteur pipette and diluted 1: 1 in a serum free medium. Incubation was done in $2 \times 10^{6}$ cells $/ \mathrm{ml}$ of the medium, in DMEM/F12/10FB: $50 / 50$ 
DMEM/Ham's F12 with $10 \%$ fetal bovine serum

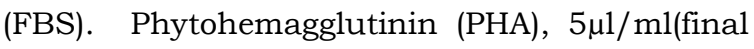
concentration) was added to stimulate mitosis from 24 to $72 \mathrm{~h}$. Sample was collected at 24, 36, 48,60 , and $72 \mathrm{~h}$, and smears were prepared to determine the incubation time. $0.001 \mu \mathrm{g} / \mathrm{ml}$ (final concentration) of Colecemid was added for $2 \mathrm{~h}$ and cells were centrifuged after the Colecemid treatment, pellets were resuspended in $0.075 \mathrm{M}$ KCL for hypotonic swelling, processed for the smear preparation and stained with Giemsa stain.

\section{RESULT \& DISCUSSION:}

In Clarias gariepinus, metaphase chromosome number was observed to be $2 \mathrm{n}=54$ (Fig.1). Among 54 chromosomes, 8 metacentric, 22 submetric, 14 subtelocentric and 10 were telocentric. Range of the length of the chromosomes was from $4.96 \mu$ to $1.92 \mu$ (Fig.2, 3) . In C. gariepinus 24 homomorpic pairs of chromosomes containing 4 pairs of metacentric(M) (numbers 23,24,25, $26,45,49,50), 11$ pairs of submetacentric (SM) (numbers, $1,2,3,4,5,6,7,8,9,10,11, \quad 12, \quad 13$, $14,17,18, \quad 19, \quad 20,27,28,39,40), \quad 7$ pairs of subtelocentric (ST) (numbers 21, 22, 29, 30, 31, $32,33,34,41,42,43,44,51,52)$ and 5 pairs of telocentric (T) (numbers 15,16, 35, 36, 37, 38, 47, $48,53,54$ ) (Fig. 2) leading to the chromosomal formula of $\mathrm{N}=4 \mathrm{M}+11 \mathrm{SM}+7 \mathrm{ST}+5 \mathrm{~T}$ were identified.

In the present karyotypic study Clarias gariepinus shows $2 \mathrm{n}=54$ number of chromosomes but in Clarias batrachus $2 \mathrm{n}=52$ or $2 \mathrm{n}=50$ and in Heteropneustes fossilis $2 \mathrm{n}=58$ or $2 n=56$ number of chromosomes as reported by Vasudevan et al. (1973) and Datta-Munshi and Hughes (1992). Diploid number in fishes ranges from 12 or 16 to 239 . The highest number of 239 has been recorded in Acipencer naccarii ( Fontana and Colombo, 1947). In C. batrachus, chromosomal formula is $18 \mathrm{M}+20 \mathrm{SM}+8 \mathrm{ST}+4 \mathrm{~T}$, in $H$. fossilis chromosomal formula is
$14 \mathrm{M}+26 \mathrm{SM}+16 \mathrm{ST}+00 \mathrm{~T}$ (Datta-Munshi and Hughes, 1992) and in C. gariepinus chromosomal formula is worked out as $8 \mathrm{M}+20 \mathrm{SM}+12 \mathrm{ST}+14 \mathrm{~T}$.

\section{CONCLUSION:}

Thus species-specific differences in chromosome number, type of chromosomes and their pattern is observed. In carps, number of chromosomes is almost similar throughout different species and in Labeo dero 2n=54 (Khuda-Bukhsh and Chanda, 1989). For hybridization of fish, knowledge of karyotype of chromosome is important. Such work is carried out in carps (Manna, 1983) but such studies are lacking in catfishes, so present attempt is made to explore this knowledge.

\section{REFERENCES:}

Cucchi, C. and Baruffaldi, A. (1990). A new method for karyological studies in teleost fishes. J. Fish Biol., 3: 71-75

Datta-Munshi, J.S. and Hughes, G.M. (1992). Air breathing Fishes of India Their structure, Function and Life History. OXFORD \& IBH PUBLISHING CO. PVT. LTD. New Delhi.

Fontana, F. and Colombo, G. (1947) Experientia, 30: 739 .

Kligerman, A.D. and Bloom, S.E. (1977). Rapid chromosome preparation from solid tissue of fish. J. Fish. Res. Board Can., 23: 767-769.

Khuda-Bukhsh, A.R. and Chanda, T. (1989). Somatic chromosomes of three species of hillstream fishes from Assam. In: Fish Genetics in India, (Eds. Das, P. \& Jhingran, A.G.). Today \& Tommorow's Printers \& Publishers, New Delhi. p.p. 69-73.

Manna, G.K.(1983). Cytogenetics studies on fishes and amphibia. Proc.XV Intnatl Congr. Genet, New Delhi. In: Genetical research in India. ICAR, Pub. NND. New Delhi. p.p. 244273. 
Mohanty, M.K. (2004). A report on the karyotype and NORs of A passerine bird. Perspectives in Cytology and Genetics, 11: 215-218.
Vasudevan, P., Rao,S.G.A. and Rao, S.R.V. (1973):Somatic and Meiotic chromosomes of Heteropneustes fossilis . Curr. Sci., 42(12);427-428.

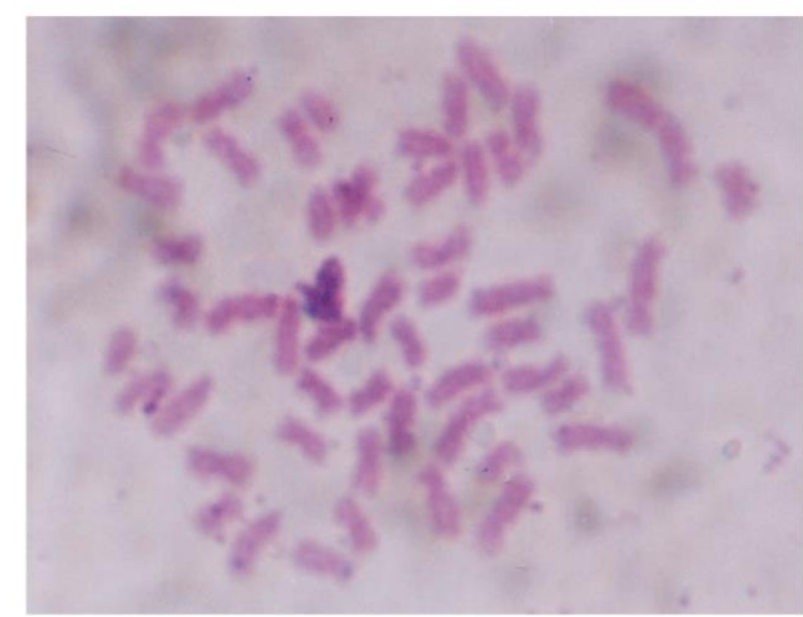

Fig.1 Metaphase in Clarias gariepinus showing 54 chromosomes

$1800 X$

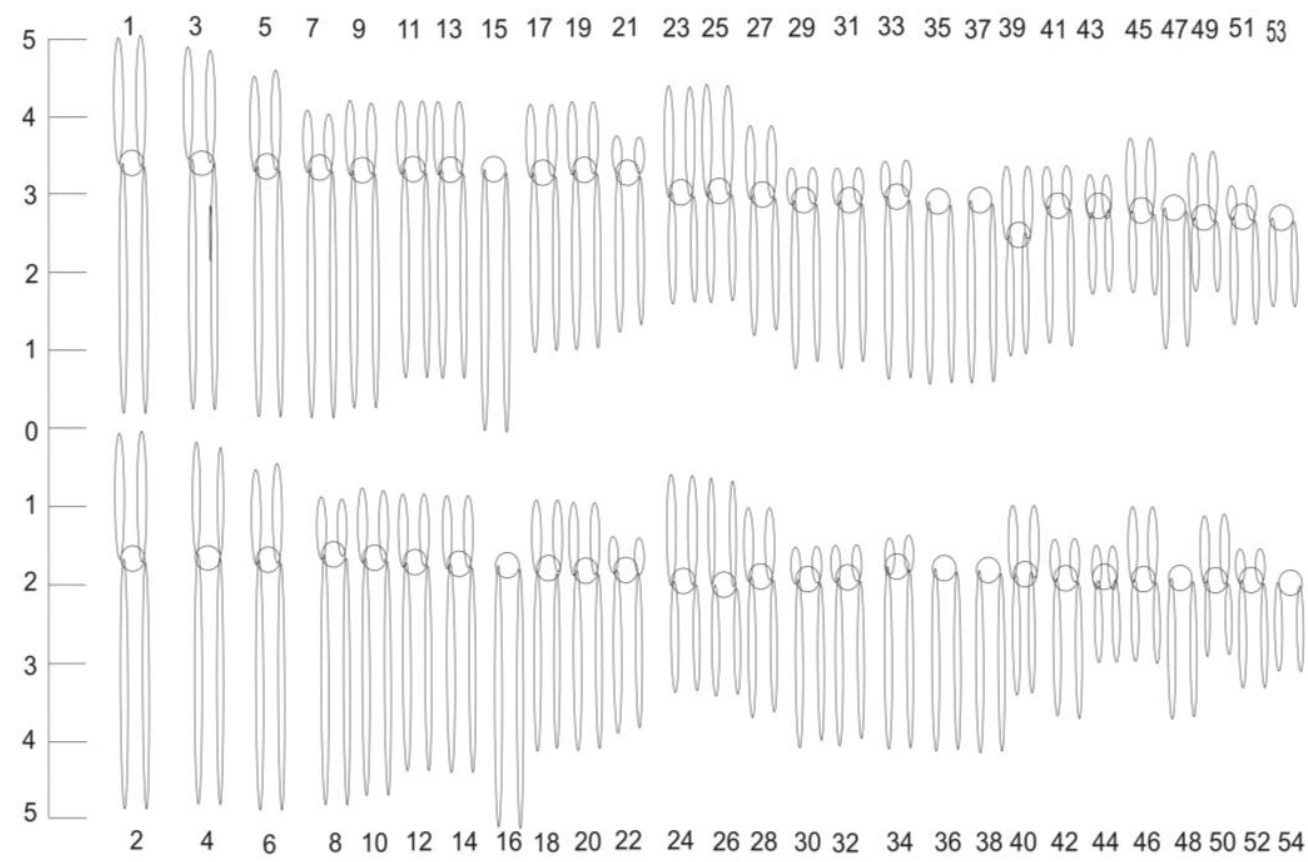

Fig. 2 Diagrammatic representation of idiogram of Clarias gariepinus $1 \mathrm{div}=1 \mu$ 


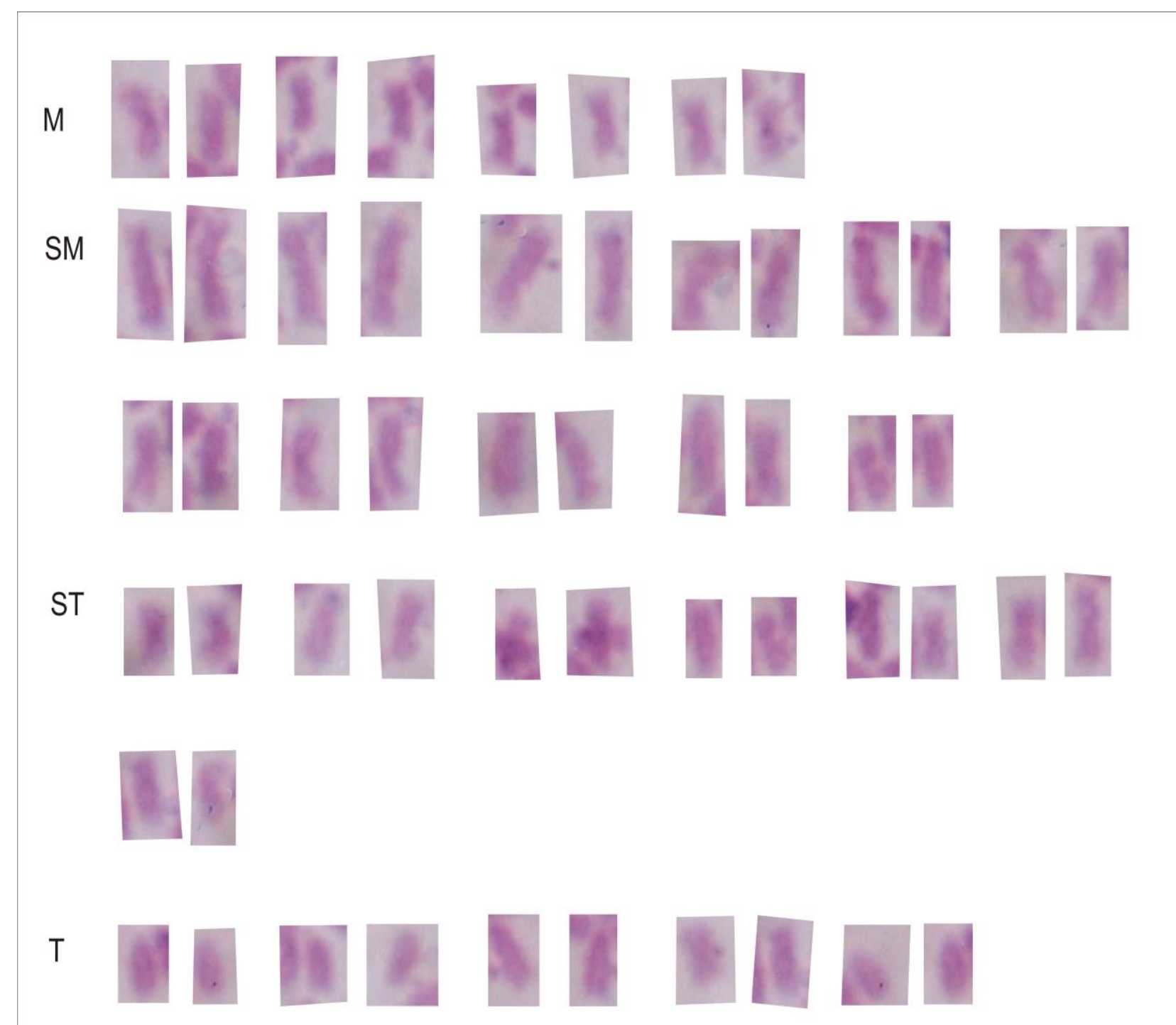

Fig. 3 Karyotype of diploid chromosome of Clarias gariepinus (M-Metacetric, SM -Submetacentric, ST-Subtelocentric and T- Telocentric) X 1800 